\title{
DIABETES
}

\section{Circulating factors implicated in diabetic enteropathy}

According to new results published in Cell Stem Cell, altered levels of circulating serum factors in patients with type 1 diabetes mellitus (T1DM) results in abnormal intestinal mucosa morphology. The survival of colonic stem cells (CoSCs) was negatively affected by decreased levels of insulin-like growth factor 1 (IGF-1) and increased levels of insulin-like growth factor binding protein 3 (IGFBP-3).

Diabetic enteropathy affects up to $70 \%$ of patients with T1DM, but the underlying mechanisms are poorly defined. However, intestinal homeostasis is dependent on the proliferation and differentiation of CoSCs, located in the intestinal crypts. "We aimed to unveil the mechanisms behind diabetic enteropathy development and whether stem cells and circulating factors were somehow involved," explains corresponding author Paolo Fiorina.

Diarrhoea, abdominal pain and constipation were observed in patients with T1DM. Analysis of the intestinal mucosa revealed reduced crypt numbers

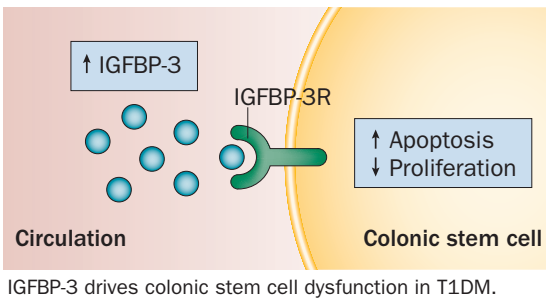

and a substantial decrease in epithelialcell proliferation. A subsequent screen of circulating serum factors showed reduced levels of IGF-1 and increased levels of IGFBP-3 in patients with T1DM compared with healthy individuals.

Crypts isolated from patients with T1DM and cultured to form organoids failed to grow, unlike those cultured from healthy people. The investigators showed that addition of IGF-1 to the culture medium rescued organoid growth, but addition of IGFBP-3 negated this improvement.

IGFBP-3 was found to promote apoptosis by directly binding the IGFBP-3 receptor (IGFBP-3R, also known as TMEM219).
Knockdown of IGFBP-3R protected the proliferation and self-renewal of organoids when exposed to IGFBP-3. The authors found that in mice with diabetic enteropathy, administration of IGFBP-3 enhanced the reduction in crypt numbers and CoSCs seen in untreated mice. IGF-1 treatment only partially improved the number of crypts and CoSCs.

Finally, Fiorina and colleagues generated a recombinant protein based on the IGFBP-3R extracellular domain. Treatment with this protein preserved crypt growth and prevented CoSC apoptosis in crypt organoids cultured with IGFBP-3 and improved mucosal morphology in diabetic mice.

\section{Hugh Thomas}

This article has also been published in Nat. Rev. Endocrinol. (doi:10.1038/nrendo.2015.184).

Original article D'Addio, F. et al. Circulating IGF-I and IGFBP3 levels control human colonic stem cell function and are disrupted in diabetic enteropathy. Cell Stem Cell doi:10.1016/j.stem.2015.07.010 